\title{
Evaluation of Aging in Air of Poly (Ethylene Terephthalat) in Oxygen Plasma
}

\author{
Michelle Cequeira Feitor ${ }^{a}$, Clodomiro Alves Junior ${ }^{b}$, Clovis Medeiros Bezerra ${ }^{a}$, \\ Rômulo Ribeiro Magalhães de Sousa ${ }^{c}$, Thercio Henrique de Carvalho Costa ${ }^{b *}$
}

\begin{abstract}
${ }^{a}$ Departamento de Engenharia Têxtil, Universidade Federal do Rio Grande do Norte - UFRN, Av. Sem. Salgado Filho, Campus Universitário, Lagoa Nova, CP 1524, CEP 59072-970 Natal, RN, Brazil ${ }^{b}$ Departamento de Engenharia Mecânica, Universidade Federal do Rio Grande do Norte - UFRN, Av. Sem. Salgado Filho, Campus Universitário, Lagoa Nova, CP 1524, CEP 59072-970, Natal, RN, Brazil ${ }^{c}$ Departamento de Engenharia Mecânica, Universidade Federal do Piauí-UFPI, Av. Ininga, s/n, Campus Universitário Ministro Petrônio Portella, Ininga, CEP 64049-550, Teresina, PI, Brazil
\end{abstract}

Received: July 1, 2014; Revised: August 31, 2015

\begin{abstract}
Polyester fabric samples - PET (poly (ethylene terephtalate)), were treated with oxygen plasma, in order to alter the hydrophilicity of such material. The process parameters: working pressure, current, tension and temperature were kept constant, varying only the treatment time. In order to evaluate the change caused on samples hydrophilicity, as well as the influence of the treatment time, the vertical wicking test was used. The samples were stored at standard temperature and pressure conditions, and their wettability were measured one day after the treatment and repeated after 30,60 and 360 days, in order to check the stability of the effects produced. Scanning electron microscopy (SEM), Raman and ATR (Attenuated total reflection) spectroscopies were used to evaluate physical and chemical alterations on the samples surface. The results have shown a substantial improvement on the hydrophilicity of the treated samples compared to the non-treated sample.
\end{abstract}

Keywords: aging treatment, low-temperature plasma nitriding, vertical wicking, wettability

\section{Introduction}

Polymers are materials of large industrial application. However their properties like hydrophilicity; biocompatibility, adhesion, friction and dyeability do not serve, eventually, the industry desires. Therefore, additional surface modification of these materials is needed to alter their properties ${ }^{1-4}$.

Fabric water absorption capacity is an important characteristic that should be fulfilled by a textile materials used in clothing, which depend mainly on its fiber properties ${ }^{5}$.

The poly (ethylene terephtalate) (PET) is used by the textile clothing industry due to its versatility and durability, though it is uncomfortable when in contact with the skin mainly due to its low water absorption capacity ( 0.2 to $0.8 \%$ ), which is due to its fiber smooth surface and absence of chemical hydrophilic groups, see Scheme 1, like $-\mathrm{OH}$ in its molecular structure ${ }^{5,6}$.

There are several techniques used to modify textile materials surface, and those involve the use of lots of water and chemical reagents, which in its majority are toxic and harmful to the environment. Low energy plasma interaction with the material occurs only on superficial level, so it only modifies a nanometric surface layer of the material and do not modify the bulk propriety of the material ${ }^{7,8}$. Therefore plasma technique is an important alternative to treat the surface of the textile materials, as it eliminates processing with water and use of chemical reagents ${ }^{9-12}$.

Low temperature plasma is produced by an electrical discharge in gases at low pressure. It consists of a mixture

*e-mail: thercioc@hotmail.com of highly reactive species like ions, radicals, electrons, and excited molecules, preserving the electrical neutrality. Its chemical composition and physical characteristics are determined by the process parameters, such as: composition of atmosphere, vacuum chamber geometry, gas pressure, gas flow rate and electrical power ${ }^{13,14}$.

Several phenomena may occur when the active plasma species reaches the material surface under treatment. The plasma process efficiency depends on the gas used, reactor working pressure, temperature and time of treatment, as well as the applied tension and also on the kind of material to be treated ${ }^{3}$. The main reactions produced by plasma treatment of polymers are surface etching and addition of new chemical groups to the surface. The etching reactions occur due to polymeric chain degradation, thus altering the surface topography. Addiction reactions occur due to recombination of carbon radicals on the polymer surface with the plasma active species, like atoms/ions of oxygen and nitrogen. Plasma of argon, oxygen, nitrogen and ammonia $\left(\mathrm{NH}_{3}\right)$ are used to modify PET's surface in order to make it hydrophilic ${ }^{3,15-17}$.

Others papers, reported that the hydrophilicity reduction of a polymeric material, after plasma treatment, is due to migration of added chemical polar groups from the polymer surface to its interior. This migration is difficult when, during the treatment, cross links are formed between the polymeric chains, resulting that the treated polymer may return to its hydrophobic original characteristic after same elapsed time ${ }^{17-22}$. 
The purpose of this work was to study the increasing of the wettability of $(100 \%)$ polyester fabrics by plasma treatment and to observe the aging of treated samples during one year. So, it was used $100 \% \mathrm{O}_{2}$ as the precursor gas altering only the time of treatment and all the others parameters like voltage, pressure, flow, current and temperature were kept constant. After treatment the wettability, topography and the chemical composition of the samples surface were analyzed. The wettability was measured through a capillarity test which was developed at the laboratory. The topography was observed through a scanning electron microscopy (SEM) and the chemical composition was studied using Attenuated Total Reflection (ATR-FTIR) Fourier Transform Infrared and Raman spectroscopies.

\section{Material and Methods}

The substrate used in this work was a $100 \%$ polyester (PET) fabric with $160 \mathrm{~g} / \mathrm{m}^{2}$, plain geometry and net form. Although clean, the fabric was washed in a bath of water and neutral detergent under agitation, at $100{ }^{\circ} \mathrm{C}$ for 10 minutes in order to remove any impurity from the production process andor storage. Then, the fabric was rinsed to guarantee complete detergent removal, dried at room temperature and finally cut in rectangular shape measuring $25 \mathrm{~mm} \times 200 \mathrm{~mm}$.

The plasma apparatus (presented schematically in Figure 1) used in this work is constituted by a continuous power source of $1 \mathrm{~kW}$, connected to the reactor. This source had a maximum voltage of $900 \mathrm{~V}$.

The reactor was composed by a vacuum chamber with glass walls, about five liters in volume, and closed by two stainless steel flanges fitted with welded fluid and gas feedthroughs. The superior flange was grounded and contains the gas filling system whose flow was regulated by a flow controller. The inferior flange is free of electrical connections, assuming the floating potential condition during the process, and its central feedthrough is used to place the energized electrode which worked as a cathode. The chamber is evacuated to approximately $7 \times 10^{-3} \mathrm{mbar}$, using a rotary vane vacuum pump. The chamber pressure was measured by a barometer, type capacitive membrane, and the cathode temperature is measured by a thermocouple of alumel-cromel, fitted inside the electrode. As samples were treated with oxygen plasma treatment rate varying from 10 to 60 minutes at a ratio of 10 minutes. Some process parameters were kept constant in all treatment as pressure 1.25 mbar current of $0.1 \mathrm{~A}$, voltage of $470 \mathrm{~V}$ and $90^{\circ} \mathrm{C}$ without thermocouple.

In order to avoid samples damage that could be attributed to the thermal energy dissipated by the cathode, the samples were placed at a distance of $70 \mathrm{~mm}$ from it, where the temperature was approximately $90{ }^{\circ} \mathrm{C}$.

\section{Samples Characterization}

The vertical wicking test, Scanning Electron Microscopy - SEM, Raman and Attenuated Total Reflection (ATR-FTIR) Spectroscopy were used to characterization and evaluation of the plasma treatment applied to the samples.

\subsection{The vertical wicking test}

The vertical wicking test was used to evaluate changes on samples hydrophilicity. It is a simple test of capillarity, on which a sample measuring $25 \mathrm{~mm} \times 200 \mathrm{~mm}$, has one of its end suspended, while $20 \mathrm{~mm}$ on the other end is immersed in a colored solution (Figure 2). A chronometer is used to measure the solution ascending time. The dye used to facilitate the visualization of the ascending liquid is a solution prepared dissolving $1 \mathrm{~g}$ of reactive yellow dye in one liter of distilled water at room temperature ${ }^{23}$.

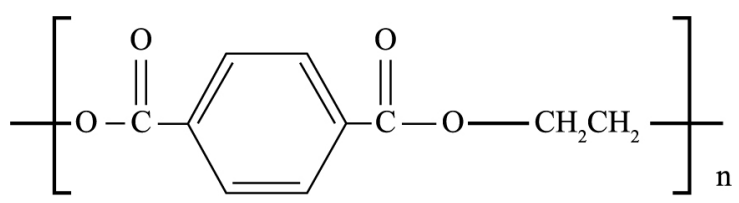

PET

Scheme 1. Molecular structure of PET.

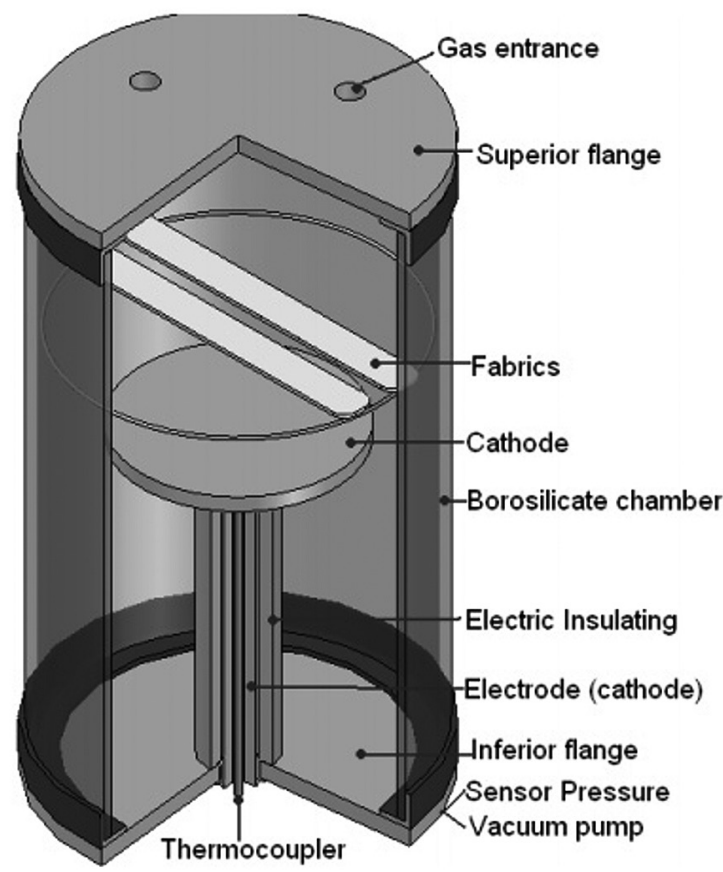

Figure 1. Schematic representation of plasma reactor.

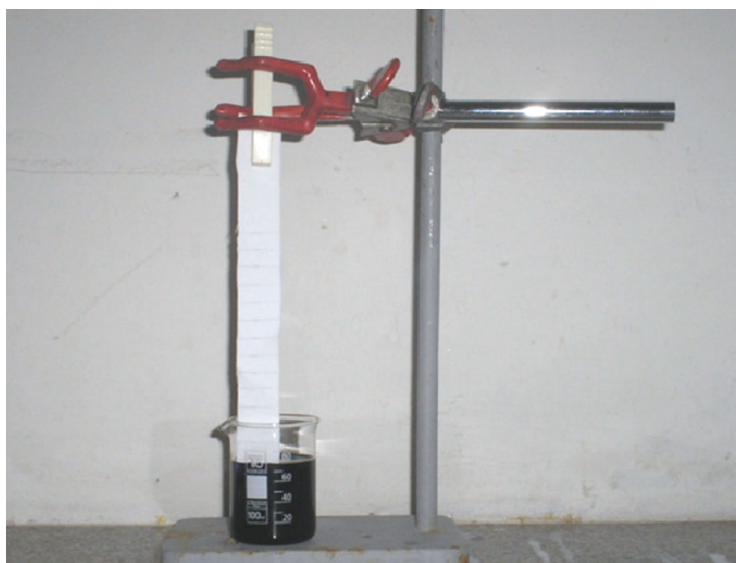

Figure 2. Illustration of the capillary test. 


\subsection{Scanning Electron Microscopy - SEM}

A scanning electron microscope (Phillips model XL-30-ESEM) was used to carry out the analysis to identify morphological alterations caused by the plasma treatment on samples surface. Some fibers were removed from the samples, and then fixed on the equipment stage with a carbon adhesive tape. The fibers were coated by a thin gold film to work as the conducting coating since the samples are dielectric.

\subsection{Attenuated Total Reflectance (ATR) and Raman spectroscopy}

A non-treated (reference sample) and the plasma treated samples were analyzed using ATR-FTIR and Raman spectroscopies in order to identify any chemical alteration occurred caused by the treatments.

\section{Results and Discussions}

\subsection{The vertical wicking test}

Figure 3 presents the results of the vertical wicking test for the treated samples as well as the standard one. The standard sample behaved as expected presenting a constant hydrophilicity, almost equal to zero. The results presented on Figure $3 \mathrm{a}$, were conducted immediately after the samples treatment. It can be observed that in the 30 first seconds, the behavior of the ascending colored solution was nearly the same for all samples, becoming different afterwards. The maximum height of the wicking $(19 \mathrm{~cm})$ was reached by the sample treated for $60 \mathrm{~min}$, and the minimum height of the wicking $(16 \mathrm{~cm})$ by the samples treated for 10 and $30 \mathrm{~min}$.

According to Rodrigues ${ }^{24}$, a fabric sample is considered hydrophilic if in the vertical wicking test the solution reaches a minimum height of $5 \mathrm{~cm}$ in 5 minutes ${ }^{23,25,26}$. Thus, all treated samples presented a substantial improvement on its hydrophilic character, since the minimum height reached was $16 \mathrm{~cm}$. Furthermore, it also became evident that the sample hydrophilic capacity increases with the increasing of the plasma treatment time.

In order to investigate the ageing effect of the applied plasma treatment, and consequently of the hydrophilicity of the samples, these were submitted again to the vertical wicking test after periods of 30,60 and 360 days of storage. The results of these tests are presented in Figure $3 b-d$, respectively. As can be seen from these figures, the samples still present a hydrophilic capacity superior to the reference

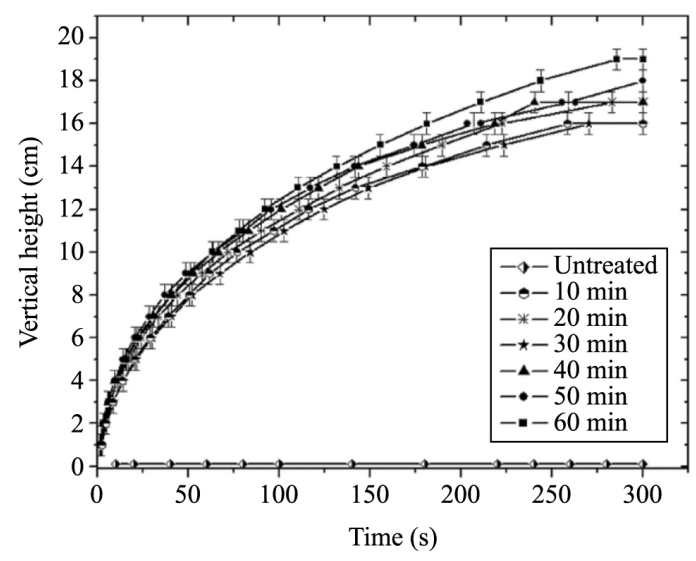

(a)

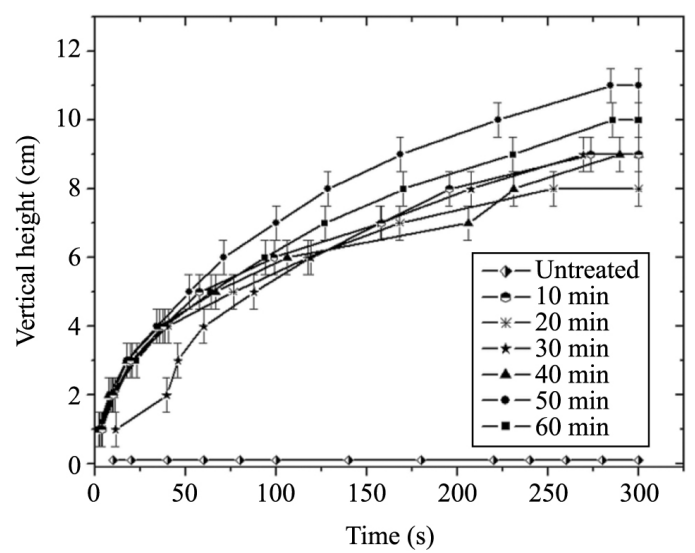

(c)

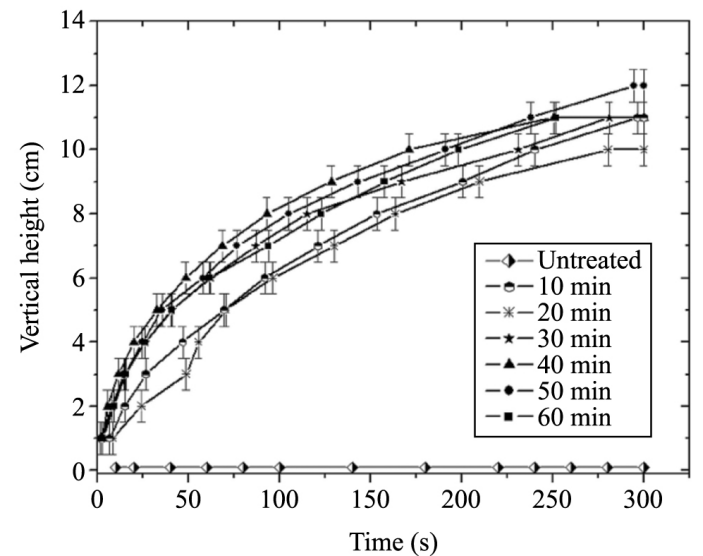

(b)

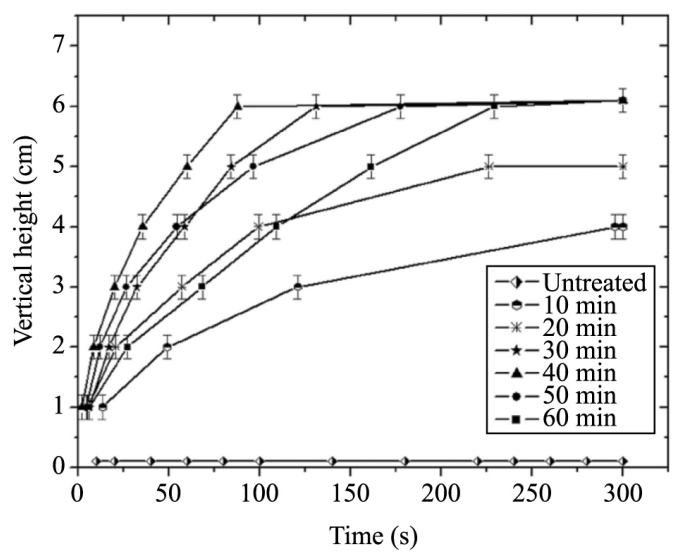

(d)

Figure 3. Results of the vertical wicking test from the treated samples and the standard one, which tests were conducted (a) immediately after the plasma treatment; (b) after a 30 days period; (c) after a 60 days; (d) after 360 days. 
sample. However, when compared to Figure $3 a$, it can be observed a drop in the hydrophilic capacity of all aged samples.

The degradation of the effect produced by the plasma treatment as a function of the storage time becomes evident in the analysis of thee results presented in Figure 4, where the colored solution maximum height values for each sample are

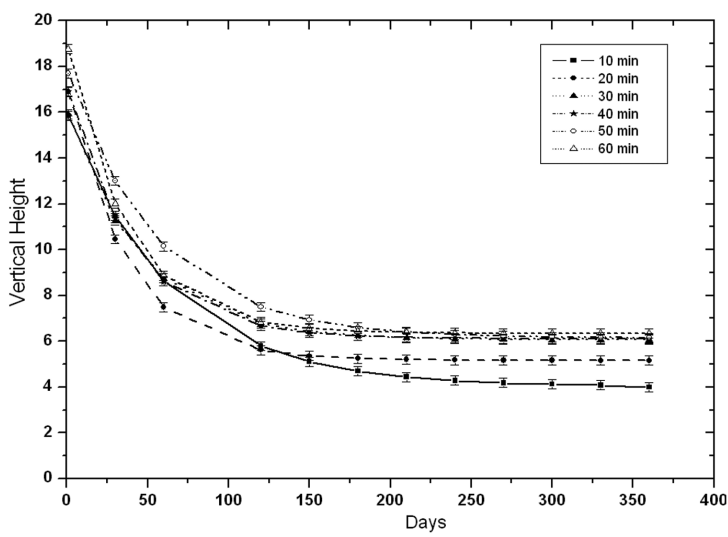

Figure 4. The effect produced by the plasma treatment as a function of the storage time.

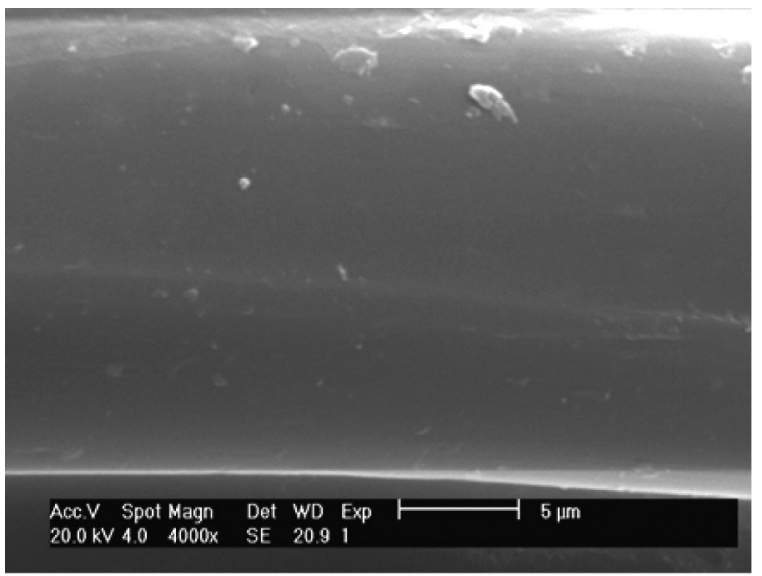

(a)

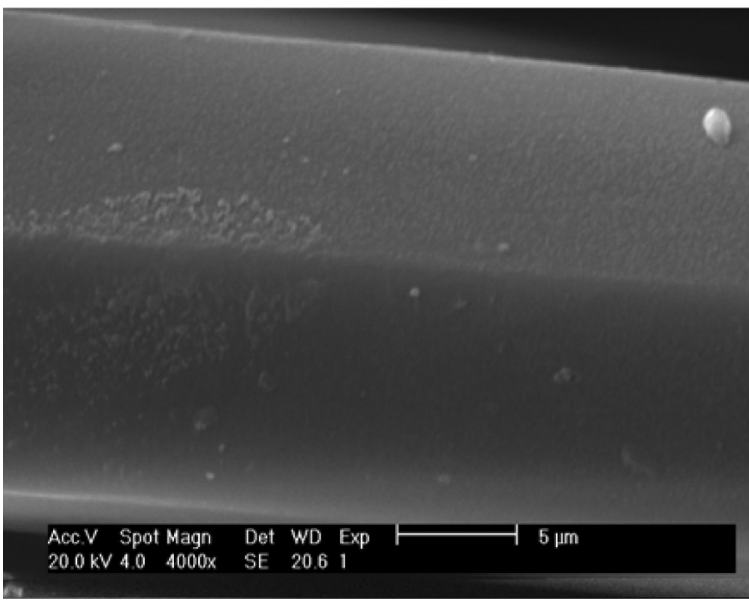

(c) plotted as a function of the time elapsed after the treatment. It can be observed that the ageing effect is accentuated in the 60 first days and then it gradually tends to stabilization. Although a substantial reduction on the solution maximum height occurred, all the treated samples, except that one treated for $10 \mathrm{~min}$, can be still classified as hydrophilic.

\subsection{Scanning Electron Microscopy - SEM}

Figure 5a-d are SEM micrographs of the fibers removed from the samples untreated and treated by 10, 30 and 60 minutes, respectively. Figure 5 a presents the image of a fiber with uniform and smooth surface, as it was expected for the non treated polyester fabric sample. Figure $5 \mathrm{~b}$ does not reveal any significant physical surface alteration, looking pretty much the same as Figure 5a. On Figure 5c it can be seen a discrete physical surface alteration (marked region). Figure $5 \mathrm{~d}$ shows that a violent attack occurred on the fiber surface, promoting substantial physical alteration on its surface. There were formations of deep erosions, which can be attributed to the break of molecular chains, due to reactions with plasma excited species on the polymer surface, as well as to the ultraviolet radiation present in the plasma.

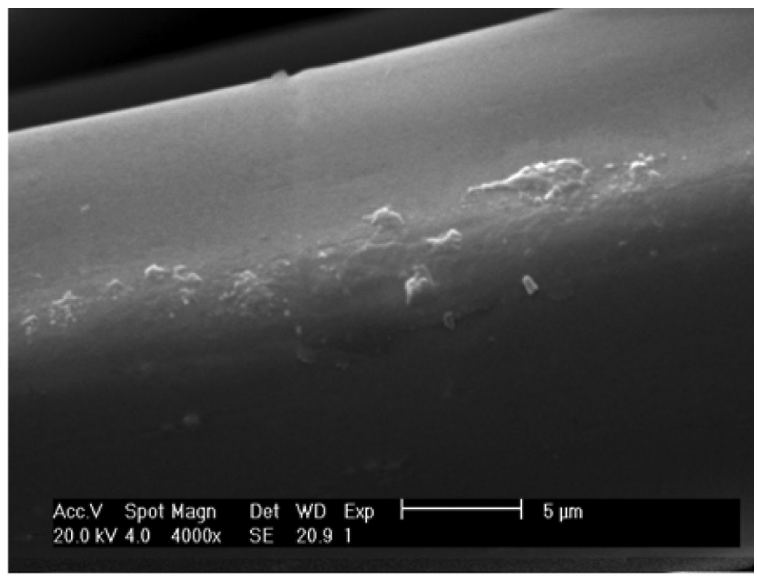

(b)

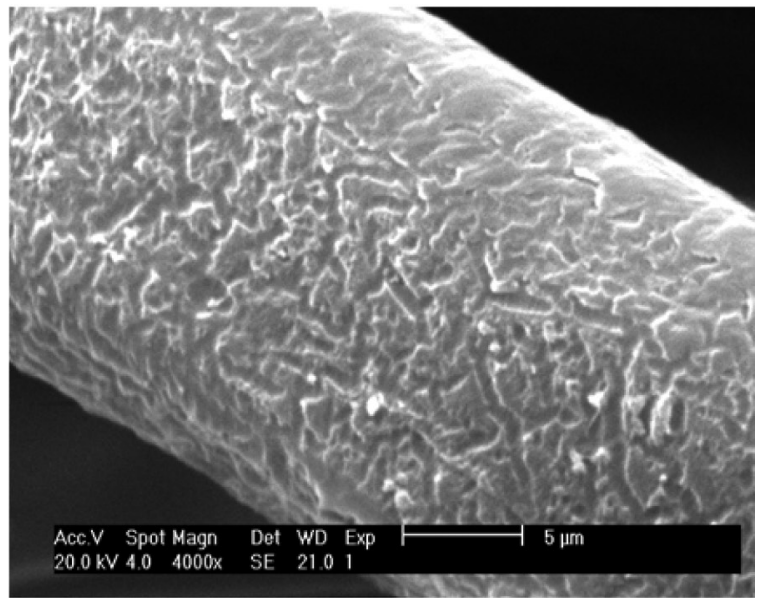

(d)

Figure 5. Fibers microscopic image of samples (a) untreated; (b) treated by 10 minutes; (c) treated by 30 minutes (d) treated by 60 minutes. 


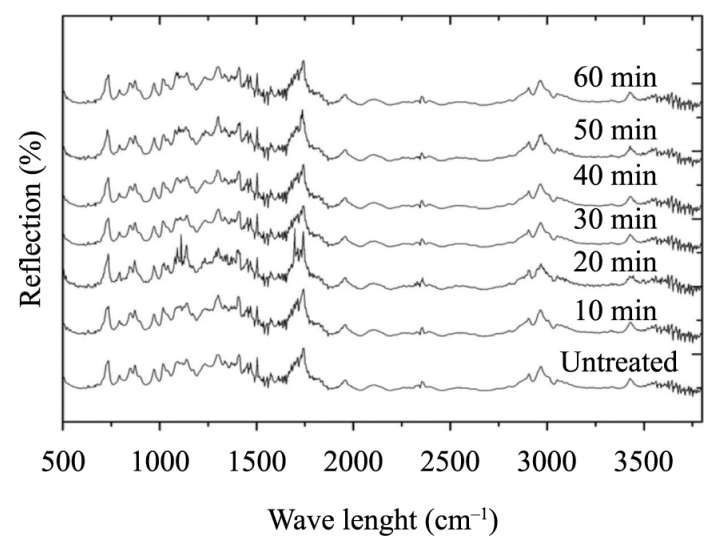

Figure 6. ATR Spectra of the treated and references samples.

Although all the treated samples presented improved hydrophilicity, only the sample treated by 60 minutes presented substantial physical alteration on its surface. This isolated fact can be used to explain the increasing in hydrophilicity of this sample, which can be attributed to the increment of fibers surface area, due to formation of erosions. However, as the other treated samples also presented increasing of hydrophilicity without demonstrating perceptible surface physical modification, it can be concluded that some chemical alteration occurred on the surface of these plasma treated samples.

\subsection{Attenuated Total Reflectance Spectroscopy (ATR) and Raman spectroscopy}

The ATR and Raman spectra of the treated and reference samples are presented in Figures 6 and 7, respectively. Comparing the spectra of the reference and the treated samples in these figures, it can not be observed formation or disappearance of any characteristic pick, which could be used to identify breaking or formation of chemical bonds, as well as addition or subtraction of chemical functional groups.

In ATR spectroscopy, reflectance measurements from the samples surface are used. However, when the incident radiation beam reaches the samples surface, it propagates some micrometers $(\mu \mathrm{m})$ into the material and is reflected back and analyzed. Thus, the reflected beam undergoes interference from the samples interior, and does not represent exclusive analysis of its surface. As the plasma treatment occurs on samples surface and only penetrates a few nanometers ( $\mathrm{nm}$ ) in its interior, this technique was proved inadequate to detect the possible chemical alterations that may have occurred.

\section{References}

1. Caiazzo F, Canonico P, Nigro R and Tagliaferri V. Electrode discharge for plasma surface treatment of polymeric materials. Journal of Materials Processing Technology. 1996; 58(1):9699. http://dx.doi.org/10.1016/0924-0136(95)02112-4.

2. Kan CW and Yuen CWM. Effect of atmospheric pressure plasma treatment on wettability and dryability of synthetic textile fibres. Surface and Coatings Technology. 2013; 228(Suppl 1):S607-S610. http://dx.doi.org/10.1016/j.surfcoat.2011.10.061.

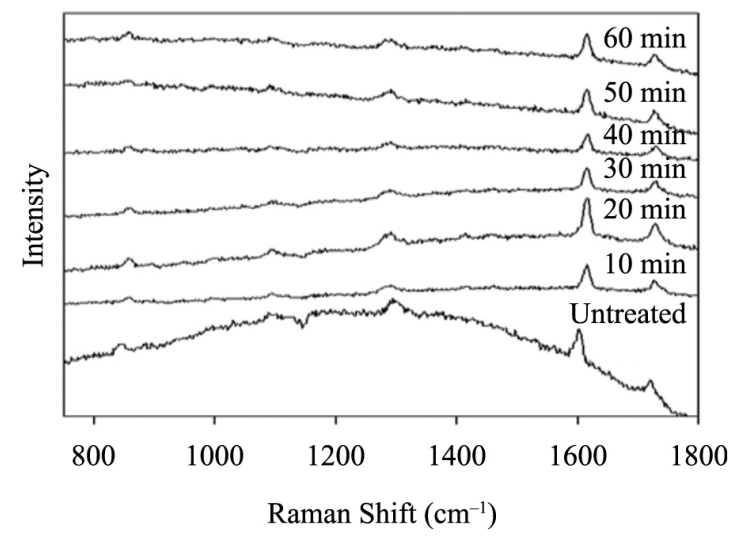

Figure 7. Raman Spectra of the treated and reference samples.

The Raman spectra, Figure 7, of the treated samples, are different when compared to that one of the untreated sample, which has a curved form, while the others present a linear form, that is, the frequency response intensities are different. This suggests a possible chemical modification on the treated samples, although it have not been characterized.

\section{Conclusion}

The plasma treatment applied to the samples in this study was proved adequate to enhance the hydrophilicity of PET hydrophobic fabric. Although the results have shown an accentuated reduction of the samples hydrophilicity on the 60 first days, the hydrophilicity became stable afterwards.

It became evident that increasing the samples treatment time conducts to more and more intense physical alterations on their surfaces. This gradually provokes surface erosion, increasing the contact area of the samples and improving the hydrophilicity. Although some chemical modifications might occur on samples surface, it was not possible to characterize them using ATR and Raman spectroscopy.

\section{Acknowledgements}

The authors would like to acknowledge the help of Dr $^{\mathrm{a}}$. Naira Maria Balzaretti, Dr. Carlos Alberto dos Santos, Dr. José Luis da Fonseca, Dra . Márcia Rodrigues and Dr. João Batista Marimon da Cunha for the collaboration in this paper. We also thank the Brazilian agency CAPES for the postgraduate scholarships.
3. Huang C-Y, Wu J-Y, Tsai C-S, Hsieh K-H, Yeh J-T and Chen K-N. Effects of argon plasma treatment on the adhesion property of ultra high molecular weight polyethylene (UHMWPE) textile. Surface and Coatings Technology. 2013; 231:507-511. http:// dx.doi.org/10.1016/j.surfcoat.2012.04.069.

4. Riccardi C, Barni R, Selli E, Mazzone G, Massafra MR, Marcandalli B, et al. Sufarce modification of poly(ethylene terephthalate) fibers induced by radio frequency air plasma treatment. Applied Surface Science. 2003; 211(1-4):386-397. http://dx.doi.org/10.1016/S0169-4332(03)00265-4. 
5. Wiener J and Dejlová P. Wicking and wetting in textiles. AUTEX Research Journal. 2003; 3:64-71.

6. Poll HU, Schladitz U and Schreiter S. Penetration of plasma effects into textile structures. Surface and Coatings Technology. 2001; 142-144:489-493. http://dx.doi.org/10.1016/S02578972(01)01055-6.

7. Negulescu II, Despa S, Chen J, Collier BJ, Despa M, Denes A, et al. Characterizing polyester fabrics treated in electrical discharges of radio-frequency plasma. Textile Research Journal. 2000; 70(1):1-7. http://dx.doi.org/10.1177/004051750007000101.

8. Poletti G, Orsini F, Raffaele-Addamo A, Riccardi C and Selli E. Cold plasma treatment of PET fabrics: AFM surface morphology characterization. Applied Surface Science. 2003; 219(3-4):311316. http://dx.doi.org/10.1016/S0169-4332(03)00703-7.

9. Carrino L, Maroni G and Polini W. Cold plasma treatment of polypropylene surface: a study on wettability and adhesion. Journal of Materials Processing Technology. 2002; 121(23):373-382. http://dx.doi.org/10.1016/S0924-0136(01)01221-3.

10. Yip J, Chan K, Sin KM and Lau KS. Low temperature plasma-treated nylon fabrics. Journal of Materials Processing Technology. 2002; 123(1):5-12. http://dx.doi.org/10.1016/ S0924-0136(02)00024-9.

11. Zille A, Oliveira, FR, Souto, AP. Plasma treatment in textile industry. Plasma Processes and Polymers. 2014; 12(2):98-131.

12. Oktem T, Ayhan H, Seventekin N and Piskin E. Modification of polyester fabrics by in situ plasma or post-plasma polymerization of acrylic acid. Journal Society Dyers and Colorists. 1999; 115(9):274-279.

13. Costa THC, Feitor MC, Alves C Jr, Freire PB and Bezerra CM. Effects of gas composition during plasma modification of polyester fabrics. Journal of Materials Processing Technology. 2006; 173(1):40-43. http://dx.doi.org/10.1016/j.jmatprotec.2005.11.008.

14. Vohrer U, Muller M and Oehr C. Glow-discharge treatment for the modification of textiles. Surface and Coatings Technology. 1998; 98(1-3):1128-1131. http://dx.doi.org/10.1016/S02578972(97)00549-5.

15. Beake BD, Ling JSG and Leggett GJ. Scanning force microscopy investigation of poly(ethylene terephthalate) modified by argon plasma treatment. Journal of Materials Chemistry. 1998; 8(8):1735-1742. http://dx.doi.org/10.1039/a801194j.

16. Matthews SR, Hwang YJ, McCord MG and Bourham MA. Investigation into etching mechanism of polyethylene terephthalate (PET) films treated in helium and oxygenatedhelium atmospheric plasmas. Journal of Applied Polymer Science. 2004; 94(6):2383-2389. http://dx.doi.org/10.1002/ app. 21162 .

17. Hwang YJ. Characterization of atmospheric pressure plasma interactions with textile/polymer substrates. [Thesis]. Raleigh: Faculty of North Carolina State University; 2003.

18. Hegemann D, Brunner $\mathrm{H}$ and Oehr C. Plasma treatment of polymers for surface and adhesion improvement. Nuclear Intruments and Methods in Physics Research B. 2003; 208: 281-286.

19. Foerch R, Kill G and Walzak M. Plasma surface modification of polypropylene: short-term vs. long-term plasma treatment. Journal of Adhesion Science and Technology. 1993; 7(10):10771089. http://dx.doi.org/10.1163/156856193X00592.

20. Mas A, Jaaba H, Schué F, Bellu AM, Kassis C, Linton R, et al. Modification of poly(3-hydroxybutyric acid)-co-(3-hydroxyvaleric acid) film surfaces in an oxygen low pressure plasma. Macromolecular Chemistry and Physics. 1996; 197(7):23312341. http://dx.doi.org/10.1002/macp.1996.021970723.

21. Brennan W, Feast WJ, Munro HS and Walker SA. Investigation of the aging of plasma oxidized peek. Polymer. 1991; 32(8):15271530. http://dx.doi.org/10.1016/0032-3861(91)90436-M.

22. Yasuda H, Sharma A and Yasuda T. Effect of orientation and mobility of polymer molecules at surfaces on contact angle and its hysteresis. Journal of Polymer Science. Polymer Physics Edition. 1981; 19(9):1285-1291. http://dx.doi.org/10.1002/ pol.1981.180190901.

23. Minor FW, Schwartz AM, Wulkow EA and Buckles LC. The migration of liquids in textile assemblies, part II: the wicking of liquids in yarns. Textile Research Journal. 1959; 29(12):931939. http://dx.doi.org/10.1177/004051755902901201.

24. Rodrigues EC. Controle de qualidade em química têxtil métodos práticos. Rio de Janeiro: CNI: SENAI: CETIQT; 1997.

25. Ferrero F. Wettability measurements on plasma treated synthetic fabrics by capillary rise method. Polymer Testing. 2003; 22(5):571578. http://dx.doi.org/10.1016/S0142-9418(02)00153-8.

26. Akishev Y, Grushin M, Napartovich A and Trushkin N. Novel AC and DC non-thermal plasma sources for cold surface treatment of polymer films and fabrics at atmospheric pressure. Plasmas and Polymers. 2002; 7:261-289. 